\title{
Ellipsometry of a thin film between similar media
}

\author{
John Lekner \\ Department of Physics, Victoria University of Wellington, Wellington, New Zealand
}

Received October 21, 1987; accepted January 26, 1988

\begin{abstract}
The conventional formula for the ellipsometric ratio $\rho=r_{p} / r_{s}$ diverges in the limit when the dielectric constants on either side of an inhomogeneous layer become equal, $\epsilon_{1}=\epsilon_{2}$. The general case, including $\epsilon_{1}=\epsilon_{2}$, necessitates going to second order in the layer thickness. A formula is derived that includes the $\epsilon_{1}=\epsilon_{2}$ case without divergence; the predicted maximum in the imaginary part of $\rho$ when $\epsilon_{1} \approx \epsilon_{2}$ indicates that index matching (of the bounding media) can significantly increase the ellipsometric signal.
\end{abstract}

\section{INTRODUCTION}

Recent work of Beaglehole ${ }^{1}$ has brought into focus a longstanding problem in the ellipsometry of thin films. This problem is that the first-order (in the film thickness) correction to the Fresnel formulas gives a divergent result for the ellipsometric ratio $\rho=r_{p} / r_{s}$ when the bounding media have equal dielectric constants. The equality of the dielectric constants $\epsilon_{1}, \epsilon_{2}$ of the bounding media was shown some years ago $^{2}$ to give a finite $\rho$, and, in fact, a zero $\bar{\rho}$ ( $\bar{\rho}$ is defined as the value of the imaginary part of $\rho$ at the principal angle, where the real part of $\rho$ is zero). What has emerged from the calculations of Beaglehole of $\bar{\rho}$ for a uniform film is that as $\epsilon_{2}$ tends to $\epsilon_{1}$ the magnitude of $\bar{\rho}$ first increases before going to zero at $\epsilon_{1}=\epsilon_{2}$, reaching a maximum for $\epsilon_{2}$ close to $\epsilon_{1}$. Because a maximum in the magnitude of $\bar{\rho}$ is of practical impor: tance in polarization modulation ellipsometry, ${ }^{3,4}$ we have developed a theory for the general case (encompassing all of $\epsilon_{1} \neq \epsilon_{2}, \epsilon_{1} \approx \epsilon_{2}$, and $\epsilon_{1}=\epsilon_{2}$ ). This is given in Section 3 . Before that, the conventional first-order theory is reviewed in Section 2.

\section{THE FIRST-ORDER EXPRESSION FOR $\rho$,} $\epsilon_{1} \neq \epsilon_{2}$

Consider an inhomogeneous layer of thickness $\Delta z$, of dielectric function $\epsilon(z)$, bounded by media of dielectric constants $\epsilon_{1}$ and $\epsilon_{2}$. Light, of angular frequency $\omega$ and speed (in vacuum) $c$, is incident from medium 1 . In the absence of the interfacial layer, the $s$ and $p$ polarization reflection amplitudes would be

$$
r_{s 0}=\frac{q_{1}-q_{2}}{q_{1}+q_{2}}, \quad r_{p 0}=\frac{Q_{2}-Q_{1}}{Q_{2}+Q_{1}},
$$

where $q_{1}$ and $q_{2}$ are the normal components of the wave vectors in media 1 and 2 and $Q_{1}=q_{1} / \epsilon_{1}, Q_{2}=q_{2} / \epsilon_{2}$. The $q$ 's are given by

$$
q_{1}^{2}=\epsilon_{1} \frac{\omega^{2}}{c^{2}}-K^{2}, \quad q_{2}^{2}=\epsilon_{2} \frac{\omega^{2}}{c^{2}}-K^{2} .
$$

Here $K$ is the (invariant) component of the wave vectors along the interface,

$$
(c K / \omega)^{2}=\epsilon_{1} \sin ^{2} \theta_{1}=\epsilon_{2} \sin ^{2} \theta_{2},
$$

where $\theta_{1}$ and $\theta_{2}$ are the angles of incidence and transmission.

The presence of the layer modifies the Fresnel reflection amplitudes [Eq. (1)]. The modification can be expressed as a power series in the layer thickness,

$$
\begin{aligned}
& r_{s}=r_{s 0}+r_{s 1}+r_{s 2}+\ldots, \\
& r_{p}=r_{p 0}+r_{p 1}+r_{p 2}+\ldots,
\end{aligned}
$$

where subscript $n(=0,1,2, \ldots)$ denotes terms that are $n$th order in $\omega \Delta z / c$. Now

$$
r_{s 0}=\frac{q_{1}^{2}-q_{2}^{2}}{\left(q_{1}+q_{2}\right)^{2}}=\frac{\Delta \epsilon \omega^{2} / c^{2}}{\left(q_{1}+q_{2}\right)^{2}},
$$

where $\Delta \epsilon=\epsilon_{1}-\epsilon_{2}$; thus, provided that $\Delta \epsilon \neq 0, r_{s 0} \neq 0$ and

$$
\frac{r_{p}}{r_{s}}=\frac{r_{p 0}}{r_{s 0}}+\frac{r_{p 1} r_{s 0}-r_{p 0} r_{s 1}}{r_{s 0}^{2}}+\ldots
$$

Long-wave perturbation theory, 5,6 which is reviewed in Chap. 3 of Ref. 7, gives the corrections $r_{p n}$ and $r_{s n}$ to the Fresnel reflection amplitudes. The first-order corrections are

$$
\begin{aligned}
& r_{s 1}=\frac{2 i q_{1} \omega^{2} / c^{2}}{\left(q_{1}+q_{2}\right)^{2}} \lambda_{1}, \\
& r_{p 1}=\frac{2 i Q_{1}}{\left(Q_{1}+Q_{2}\right)^{2}}\left(Q_{2}{ }^{2} \lambda_{1}-\frac{K^{2}}{\epsilon_{1} \epsilon_{2}} \Lambda_{1}\right),
\end{aligned}
$$

where the integrals $\lambda_{1}$ and $\Lambda_{1}$ are the first in the sets

$$
\begin{aligned}
& \lambda_{n}=\int_{-\infty}^{\infty} \mathrm{d} z\left(\epsilon-\epsilon_{0}\right) z^{n-1}, \\
& \Lambda_{n}=\epsilon_{1} \epsilon_{2} \int_{-\infty}^{\infty} \mathrm{d} z\left(\frac{1}{\epsilon_{0}}-\frac{1}{\epsilon}\right) z^{n-1} .
\end{aligned}
$$

Here $\epsilon_{0}(z)$ is the step function representing a sharp transition between media 1 and $2, \epsilon_{0}(z)=\epsilon_{1}$ for $z<0, \epsilon_{0}(z)=\epsilon_{2}$ for $z$ $>0$. From Eqs. (6)-(8) we find after some manipulation that 


$$
r_{s 0} \frac{r_{p}}{r_{s}}=r_{p 0}-\frac{2 i Q_{1} K^{2}}{\epsilon_{1} \epsilon_{2}\left(Q_{1}+Q_{2}\right)^{2}} I_{1}+\ldots
$$

where

$$
I_{1}=\Lambda_{1}-\lambda_{1}=\int_{-\infty}^{\infty} \mathrm{d} z \frac{\left(\epsilon_{1}-\epsilon\right)\left(\epsilon-\epsilon_{2}\right)}{\epsilon} .
$$

The companion formula to Eq. (5) is

$$
r_{p 0}=\frac{\Delta \epsilon\left(\omega^{2} / c^{2}\right)}{\epsilon_{1} \epsilon_{2}\left(Q_{1}+Q_{2}\right)^{2}}\left[1-\left(\frac{c K}{\omega}\right)^{2}\left(\frac{1}{\epsilon_{1}}+\frac{1}{\epsilon_{2}}\right)\right] .
$$

On using Eqs. (5) and (13) in Eq. (11), we find that

$$
\begin{aligned}
\frac{r_{p}}{r_{s}}= & \frac{\left(q_{1}+q_{2}\right)^{2}}{\epsilon_{1} \epsilon_{2}\left(Q_{1}+Q_{2}\right)^{2}}\left\{\left[1-\left(\frac{c K}{\omega}\right)^{2}\left(\frac{1}{\epsilon_{1}}+\frac{1}{\epsilon_{2}}\right)\right]\right. \\
& \left.-2 i Q_{1}\left(\frac{c K}{\omega}\right)^{2} I_{1} / \Delta \epsilon+\ldots\right\} .
\end{aligned}
$$

The ellipsometric quantity $\bar{\rho}$ is the value of $\operatorname{Im}\left(r_{p} / r_{s}\right)$ at the angle where $\operatorname{Re}\left(r_{p} / r_{s}\right)$ is zero. To first order in the interface thickness, the real part is zero at the Brewster angle $\theta_{B}=$ $\arctan \left(\epsilon_{1} / \epsilon_{2}\right)^{1 / 2}$, at which

$$
\begin{gathered}
\left(\frac{c Q_{1}}{\omega}\right)^{2}=\left(\frac{c Q_{2}}{\omega}\right)^{2}=\frac{1}{\epsilon_{1}+\epsilon_{2}}, \quad\left(\frac{c K}{\omega}\right)^{2}=\frac{\epsilon_{1} \epsilon_{2}}{\epsilon_{1}+\epsilon_{2}}, \\
\left(\frac{c q_{1}}{\omega}\right)^{2}=\frac{\epsilon_{1}{ }^{2}}{\epsilon_{1}+\epsilon_{2}}, \quad\left(\frac{c q_{2}}{\omega}\right)^{2}=\frac{\epsilon_{2}^{2}}{\epsilon_{1}+\epsilon_{2}} .
\end{gathered}
$$

Thus

$$
\bar{\rho}=-\frac{\left(\epsilon_{1}+\epsilon_{2}\right)^{1 / 2}}{2 \Delta \epsilon} \frac{\omega}{c} I_{1}+\ldots
$$

Formulas (14) and (17), often attributed to Drude but in fact going back to Lorenz and Van Ryn (see Rayleigh ${ }^{8}$ for a derivation and reference to earlier work), clearly fail when $\epsilon_{1}$ $=\epsilon_{2}$; the apparent divergence is due to the inadmissible division by zero in Eq. (6). When $\Delta \epsilon=0$, both $r_{p 0}$ and $r_{s 0}$ are zero, and (see Ref. 2, Sec. 4)

\section{SECOND-ORDER THEORY FOR GENERAL $\Delta \epsilon$}

We will calculate $r_{p} / r_{s}$, avoiding division by $r_{s 0}$ or $r_{p 0}$ so as to include the possibility of $\epsilon_{1}=\epsilon_{2}$. We use the form

$$
\frac{r_{p}}{r_{s}}=\frac{r_{p 0}+r_{p 1}+r_{p 2}+\ldots}{r_{s 0}+r_{s 1}+r_{s 2}+\ldots} \text {. }
$$

The first-order terms $r_{p 1}$ and $r_{s 1}$ have been given above. The second-order terms are [Ref. 2, Eqs. (15) and (29)]

$$
\begin{aligned}
r_{s 2}= & \frac{-2 q_{1} \omega^{2} / c^{2}}{\left(q_{1}+q_{2}\right)^{2}}\left(2 q_{2} \lambda_{2}+\frac{\omega^{2} / c^{2}}{q_{1}+q_{2}} \lambda_{1}{ }^{2}\right), \\
r_{p 2}= & \frac{2 Q_{1} Q_{2}}{\left(Q_{1}+Q_{2}\right)^{3}}\left\{\frac{K^{4}}{Q_{2}}\left(\frac{\Lambda_{1}}{\epsilon_{1} \epsilon_{2}}\right)^{2}\right. \\
& +K^{2}\left[\left(Q_{1}-Q_{2}\right) \frac{\lambda_{1} \Lambda_{1}}{\epsilon_{1} \epsilon_{2}}+\left(Q_{1}+Q_{2}\right) J\right] \\
& \left.-Q_{1} Q_{2}{ }^{2} \lambda_{1}{ }^{2}-2\left(Q_{1}+Q_{2}\right) \frac{\omega^{2}}{c^{2}} \lambda_{2}\right\},
\end{aligned}
$$

where $J$ is related to a second-order integral invariant $j_{2}$ [Ref. 2, Eq. (B7)] by

$$
\Delta \epsilon J=j_{2}+\left(\frac{1}{\epsilon_{1}}+\frac{1}{\epsilon_{2}}\right) \lambda_{1} \Lambda_{1} .
$$

For nonabsorbing layers the first-order terms $r_{s 1}$ and $r_{p 1}$ are imaginary, and the second-order terms $r_{s 2}$ and $r_{p 2}$ are real. We multiply the numerator and denominator of Eq. (19) by the complex conjugate of the denominator. After a lengthy rearrangement of terms, the ratio $r_{p} / r_{s}$ can be expressed in terms of the three integral invariants $I_{1}, j_{2}$, and $i_{2} ; i_{2}$ is defined as

$$
i_{2}=2 \Delta \epsilon \lambda_{2}-\lambda_{1}^{2} .
$$

(These three integral invariants characterize the reflectivities $\left|r_{p}\right|^{2}$ and $\left|r_{s}\right|^{2}$ and the ellipsometric ratio $r_{p} / r_{s}$ to second order in the layer thickness, for any layer. Their properties are discussed and their functional forms are tabulated for six profiles in Secs. 3-6 of Ref. 7.) The result is

$$
\begin{aligned}
\frac{r_{p}}{r_{s}}= & \frac{\left(q_{1}+q_{2}\right)^{2}}{\epsilon_{1} \epsilon_{2}\left(Q_{1}+Q_{2}\right)^{2}} \\
& \times \frac{(\Delta \epsilon)^{2}\left[1-\left(\frac{c K}{\omega}\right)^{2}\left(\frac{1}{\epsilon_{1}}+\frac{1}{\epsilon_{2}}\right)\right]-\Delta \epsilon 2 i Q_{1}\left(\frac{c K}{\omega}\right)^{2} I_{1}+\frac{\Delta \epsilon 2 Q_{1} K^{2}(c K / \omega)^{2} I_{1}^{2}}{\epsilon_{1} \epsilon_{2}\left(Q_{1}+Q_{2}\right)}-\dot{4} q_{1} q_{2}\left[i_{2}-\frac{1}{2}\left(\frac{c K}{\omega}\right)^{2} j_{2}+\left(\frac{1}{\epsilon_{1}}+\frac{1}{\epsilon_{2}}\right) i_{2}\right]}{(\Delta \epsilon)^{2}-4 q_{1} q_{2} i_{2}} .
\end{aligned}
$$

$$
\frac{r_{p}}{r_{s}}=\frac{r_{p 1}}{r_{s 1}}+\ldots=\cos ^{2} \theta_{0}-\frac{\Lambda_{1}}{\lambda_{1}} \sin ^{2} \theta_{0}+\ldots,
$$

where $\theta_{0}$ is the common value of $\theta_{1}$ and $\theta_{2}$ when $\epsilon_{1}=\epsilon_{2}$. The value of $\bar{\rho}$ is thus zero, not infinity, to lowest order in the interface thickness. The fact that the simple theory gives a divergence as $\Delta \epsilon \rightarrow 0$, whereas the $\Delta \epsilon=0$ value is zero, suggests the existence of a maximum for small $\Delta \epsilon$. This turns out to be true, as we will see in Section 3.
When $\Delta \epsilon \neq 0$ this ratio may be expressed in conventional form as a series of terms in increasing powers of the layer thickness, agreeing with Ref. 7, Eq. (3.52). However, because we want a theory applicable to small $\Delta \epsilon$, we will keep the form of Eq. (24).

We note some general properties of Eq. (24). All angular dependence is contained in the coefficients multiplying $I_{1}$, $I_{1}{ }^{2}, i_{2}$, and $j_{2}$, the integral invariants depending only on the interfacial thickness and profile shape $\epsilon(z)$ and on the dielec- 


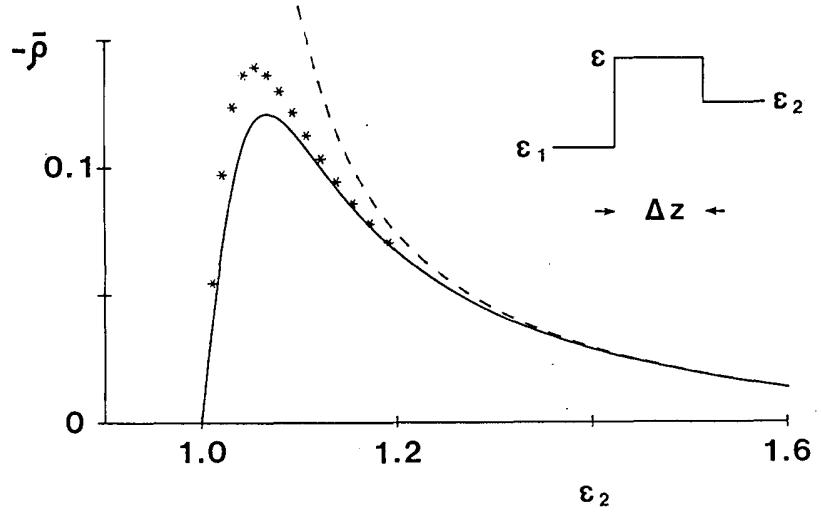

Fig. 1. The ellipsometric parameter $\bar{\rho}$ for a thin uniform film $(\omega \Delta z)$ $c=0.05, \epsilon=2$ ) between media with dielectric constants $\epsilon_{1}=1$ and variable $\epsilon_{2}$. The dashed curve is from Eq. (17), the conventional first-order theory, and diverges at $\epsilon_{2}=\epsilon_{1}$. The solid curve is from expression (25), which is an approximate version of the secondorder theory. The points are calculated from the exact reflection amplitudes. The $\bar{\rho}$ deduced from the second-order expression [Eq. (24)] is indistinguishable from the exact $\bar{\rho}$ for the small film thickness used here.

tric constants of the bounding media. At normal incidence $(K=0)$, we find $r_{p} / r_{s} \rightarrow 1$, as it must, since there is then no physical difference between the $s$ and $p$ waves. At grazing incidence $\left(q_{1}, Q_{1} \rightarrow 0\right), r_{p} / r_{s} \rightarrow-1$, in accord with a general theorem of reflection (Ref. 7, Sec. 2-3). When $\Delta \epsilon=0$ the invariants $i_{2}$ and $j_{2}$ take the values $-\lambda_{1}^{2}$ and $-2 \lambda_{1} \Lambda_{1} / \epsilon_{0}$, respectively, and $r_{p} / r_{s}$ reduces to the value given in Eq. (18). [We note in passing that Eq. (18) tends not to the correct value of -1 at grazing incidence but to $-\Lambda_{1} / \lambda_{1}$. This discrepancy arises from the divergence of perturbation theory at grazing incidence when $\epsilon_{1}=\epsilon_{2}$, a difficulty that is discussed in Sec. 3-5 of Ref. 7. The divergence is removed in variational theory, ${ }^{9}$ where for $\epsilon_{1}=\epsilon_{2}$ and near grazing incidence,

$$
r_{s}^{\mathrm{var}} \rightarrow \frac{-1}{1+\frac{2 i q_{0}}{\lambda_{1} \omega^{2} / c^{2}}}, \quad r_{p}^{\mathrm{var}} \rightarrow \frac{1}{1+\frac{2 i q_{0}}{\Lambda_{1} \omega^{2} / c^{2}}} .
$$

Thus, when $\left(\epsilon_{0}\right)^{1 / 2} \cos \theta_{0}$ is much less than $\lambda_{1} \omega / c$ and $\Lambda_{1} \omega / c$ respectively, $r_{s} \rightarrow-1$ and $r_{p} \rightarrow 1$, as required. For thin films this limit is reached, however, only for $\theta_{0}$ close to $90^{\circ}$. When $\epsilon_{1}=\epsilon_{2}$ the formula $r_{p} / r_{s} \approx \cos ^{2} \theta_{0}-\left(\Lambda_{1} / \lambda_{1}\right) \sin ^{2} \theta_{0}$, derived as in Eq. (18) or from Eq. (24), is correct to lowest order in the film thickness and accurate away from grazing incidence.]

Of particular interest is the value of $\bar{\rho}$. From Eq. (24) we see that the real part of $r_{p} / r_{s}$ is zero at an angle $\theta_{p}$ that differs in second order in the film thickness from the Brewster angle $\theta_{B}=\arctan \left(\epsilon_{2} / \epsilon_{1}\right)^{1 / 2}$. Approximating $\theta_{p}$ by $\theta_{B}$ and using Eqs. (15) and (16) in Eq. (24), we find that

$$
\bar{\rho} \approx \frac{-\frac{1}{2} \Delta \epsilon\left(\epsilon_{1}+\epsilon_{2}\right)^{1 / 2} \frac{\omega}{c} I_{1}}{(\Delta \epsilon)^{2}-\frac{4 \epsilon_{1} \epsilon_{2}}{\epsilon_{1}+\epsilon_{2}} \frac{\omega^{2}}{c^{2}} i_{2}} .
$$

This tends to zero as $\Delta \epsilon \rightarrow 0$, as required. The exact $\bar{\rho}$ (to second order, but not assuming $\theta_{p}=\theta_{B}$ ) is also zero when $\Delta \epsilon$ $=0$ because the functional form $\Delta \epsilon I_{1} /\left[(\Delta \epsilon)^{2}-4 q_{1} q_{2} i_{2}\right]$ is retained.

For a uniform layer,

$$
\begin{aligned}
& I_{1}=\frac{\left(\epsilon_{1}-\epsilon\right)\left(\epsilon-\epsilon_{2}\right)}{\epsilon} \Delta z, \quad i_{2}=\left(\epsilon_{1}-\epsilon\right)\left(\epsilon-\epsilon_{2}\right)(\Delta z)^{2}, \\
& j_{2}=\frac{2\left(\epsilon_{1}-\epsilon\right)\left(\epsilon-\epsilon_{2}\right)}{\epsilon}(\Delta z)^{2} .
\end{aligned}
$$

Figure 1 shows $\bar{\rho}$ calculated exactly [or from Eq. (24)], from expression (25) and from Eq. (17) for a thin uniform layer $(\epsilon$ $=2$ ) between media with $\epsilon_{1}=1$ and variable $\epsilon_{2}$. The thickness parameter $(\omega / c) \Delta z$ is 0.05 , which corresponds to a film thickness of approximately $5 \mathrm{~nm}$ for $\lambda_{1}=632.8 \mathrm{~nm}$.

From expressions (25) and (26) and by using the fact that the maximum magnitude of $\bar{\rho}$ occurs for $\epsilon_{1}$ near $\epsilon_{2}$, we find that the maximum magnitude occurs at

$$
\epsilon_{2}-\epsilon_{1} \approx\left(2 \epsilon_{1}\right)^{1 / 2}\left(\epsilon-\epsilon_{1}\right) \frac{\omega}{c} \Delta z
$$

and takes the value

$$
\epsilon_{2}-\epsilon_{2} \approx\left(2 \epsilon_{1}\right)^{1 / 2}\left(\epsilon-\epsilon_{1}\right) \frac{\omega}{c} \Delta z
$$

and takes the value

$$
\bar{\rho}_{m} \approx \frac{\epsilon_{1}\left(\epsilon_{1}-\epsilon\right)}{4 \epsilon} .
$$

Note that $\bar{\rho}_{m}$ is independent of the film thickness: index matching $\left(\epsilon_{2}\right.$ close to $\left.\epsilon_{1}\right)$ can give the large ellipsometric signal [expression (28)] even for very thin films.

\section{ACKNOWLEDGMENT}

It is a pleasure to acknowledge stimulating conversations with David Beaglehole.

\section{REFERENCES}

1. D. Beaglehole, "Ellipsometry of thin substrates and intruding layers," submitted by J. Opt. Soc. Am. A.

2. J. Lekner, "Invariant formulation of the reflection of long waves by interfaces," Physica 128A, 229-252 (1984).

3. R. M. A. Azzam and N. M. Bashara, Ellipsometry and Polarized Light (North-Holland, Amsterdam, 1977).

4. D. Beaglehole, "Ellipsometric study of the surface of simple liquids," Physica 100B, 163-174 (1980).

5. J. Lekner, "Reflection of long waves by interfaces," Physica 112A, 544-556 (1982)

6. J. Lekner, "Second-order ellipsometric coefficients," Physica 113A, 506-520 (1982).

7. J. Lekner, Theory of Reflection of Electromagnetic and Particle Waves (Nijhoff, Dordrecht, 1987).

8. J.W.S. Rayleigh "On the propagation of waves through a stratified medium, with special reference to the question of reflection," Proc. R. Soc. London Ser. A 86, 207-266 (1912).

9. J. Lekner, "Reflection of light by a nonuniform film between like media," J. Opt. Soc. Am. A 3, 9-15 (1986). 